\title{
The Landsat 7 imagery gap filling using median filter method
}

\author{
Wisam Abbas Faeq ${ }^{1}$ and Alaa S. Mahdi ${ }^{2}$ \\ ${ }^{1}$ Department of Physics, College of Sciences, University of Baghdad \\ ${ }^{2}$ Remote Sensing Unit, College of Sciences, University of Baghdad \\ Wiasm.luffy.w1@gmail.com \\ E-mail: wisam.luffy.wl@gmail.com
}

\begin{abstract}
The Enhanced Thematic Mapper Plus (ETM+) was launched on the Landsat-7 satellite on April 15, 1999. Four years later, the image was dramatically changed in May 2003 by ETM+ due to the failure of the Scan Line Corrector (SLC). The image contains $22 \%$ The damaged pixels. Intermediate filter is one of the important filters for correcting image noise, processing and basic construction in many image processing situations. Digital images are often distorted by pulse noise due to sensor errors, resulting in communication channel errors, such as switching signals from analog to digital. This error inevitably changes the intensity of the pixel image, while some pixels remain unchanged. To get rid of driving noise and improve the quality of the image we are working on, we will use an average filter. Landsat-7 data was corrected using a focal analysis method that includes the median filtering process. We provide a method based on a certain algorithm to apply the filter to images. We apply the median filter $(3 \times 3)$ to correct and correct the image taken by Landsat 7 , and we will restore the damaged pixels using the ERDAS imaging Software.
\end{abstract}

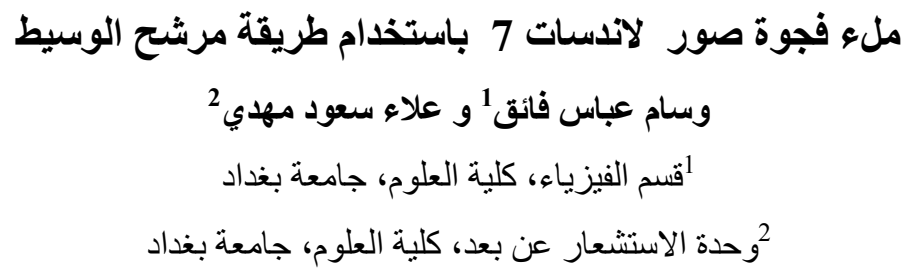

Key words

Landsat7, scan line corrector, focal analysis, median filter, ERDAS imaging.

\section{Article info.}

Received: May. 2019 Accepted: Jul. 2019

Published: Sep. 2019

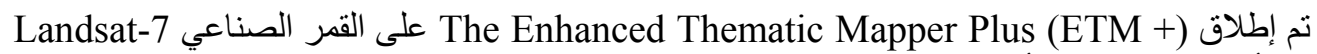

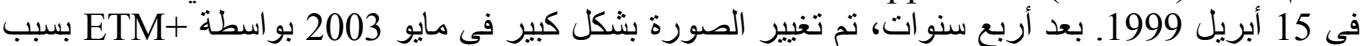

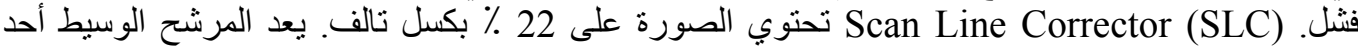

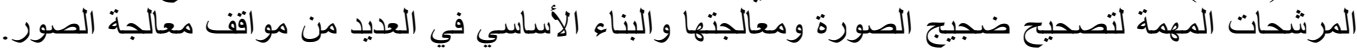

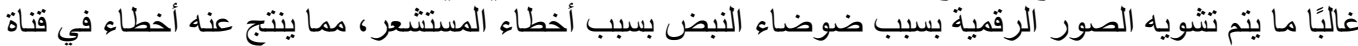

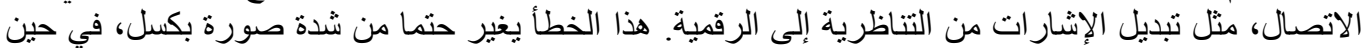

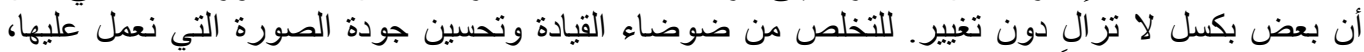

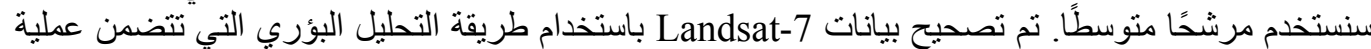

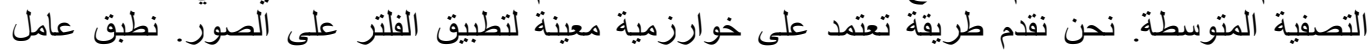

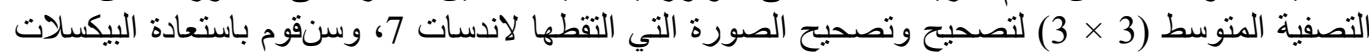

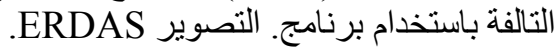




\section{Introduction}

Image processing is a modern and fast-growing field of computer science and engineering. The growth of this area has been improved by technological advances in digital signal processing devices, data storage devices, computer processors and digital computers. All regions that have traditionally worked on analog visualization are now gradually transforming into digital systems for ease of use, flexibility, and accessibility. Image processing is one of the most useful methods in areas such as medicine, remote sensing, film, video, photography, military analysis, desktop publishing, management and production automation. [1, 2]. Different applications, such as those mentioned, require sharp and bright images, so damaged or degraded images must be processed to facilitate identity recognition and continuous work. Image processing methods such as object detection, image filtering for image manipulation, and image enhancement are used, depending on the type of noise that caused a change in the intensity of some image pixels. Fig. 1 shows an example of how to calculate the median filter.

Digital image processing refers to digital image processing using the computer. The digital image consists of a limited number of elements, each with a specific value and location. Pixel is the most commonly used term for the digital image element. Image files are either pixel data or vector data that is punctuated into pixels when viewed in vector graphics. Pixels are shaped as rows and placed in a picture; each pixel contains a number that represents the intensity (color and brightness). The size of the image depends on the number and intensity of the pixels. The larger the number of pixels in a picture, the sharper the clarity and accuracy of the image, although the increase in the number of pixels increases the size of the image, the 8-bit pixel stores 256 colors, while the 16-bit pixel stores 65,536 colors, known as the real color [3].

In image processing, the distortion is corrected in picture. The Median filter is designed to solve the problem we are experiencing, the filter corrects and eliminates noise in its image. The window is selected to cover the set $(\mathrm{k} \mathrm{x}$ k) of the pixels.

$\mathrm{K}^{2}=2 \mathrm{n}+1 \rightarrow \mathrm{n}=\frac{k^{2}-1}{2}$

where, for the correct number $\mathrm{n}>0$, $\mathrm{k}=3, \quad 5, \quad 7, \quad \ldots$. In Median Filter technology, the pixels are sorted and the medium is selected [4].
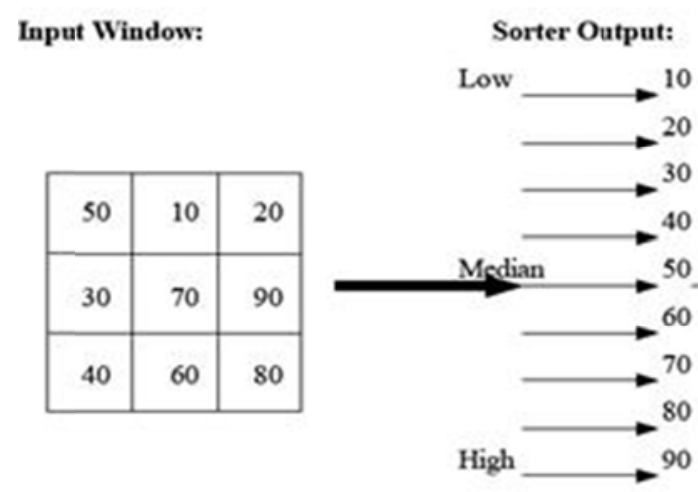

Output Pixel:

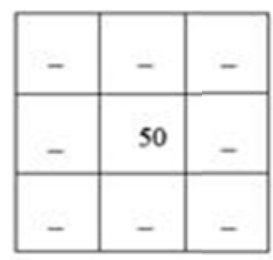

Fig. 1: a graphical depiction of the median filter operation [4]. 


\section{The Landsat-7 imagery}

Digital image filtering techniques can be divided into two large filtering processes in the field of frequency and filtering in the spatial field. The filtration process in the spatial area depends on the direct processing of the image pixels, while the filtering process in the frequency field is related to the conversion and instantaneous adjustment of the image. After May 2003, the satellite images obtained using the ETM + satellite sensor for Landsat 7 were damaged in some image pixels, which account for $22 \%$ of the original image. The shape of the zigzag line and these lines contain a loss and a clear repetition of the data that is visible along the eastern and western edges of the scene and the two gradients disappear as we approach the center of the space scene. So you need special treatment to remove their effects.

The loss and repetition in the data that is clearly visible at the edges of the satellite scene of the space scene of the Eastern and Western scenes and at least gradually fades towards the center of the scene of the scene. These images contain $22 \%$ of the black lines (SLCoff) and this requires special treatment to eliminate the effects of the Median Filter application $(3 \times 3)$ using ERFAS imagine, [5]. Erdas imagine is a Software that deals with spatial data, geometric and radiological corrections, types of improvements and spatial tests, and the development of Anaglyph technology(is the topographic toolkit of the ERDAS Software), on how to convert between vector and Raster modes.

\section{The correction method}

Focal analysis is an important process to reduce noise in the image, such as the dead sensor tape, random mutations in data sets and other image defects. It is a useful process for enhancing images.

Focal Analysis selects the area around pixels to be modified. The operations that can be performed on the selected pixel can be specified sum, Means and Broker.

Focus analysis is the process of Median filter which is taken from this algorithm:

1. Place all pixels with the moving window selected in numeric order.

2. Replace the pixel to be adjusted with the Digital Number (DN) value in the middle of the order [6].

We open a picture of Landsat 7 provided that its image has a scan line corrector (SLC) (as shown in the Fig.2) in the Erdas imagine program.

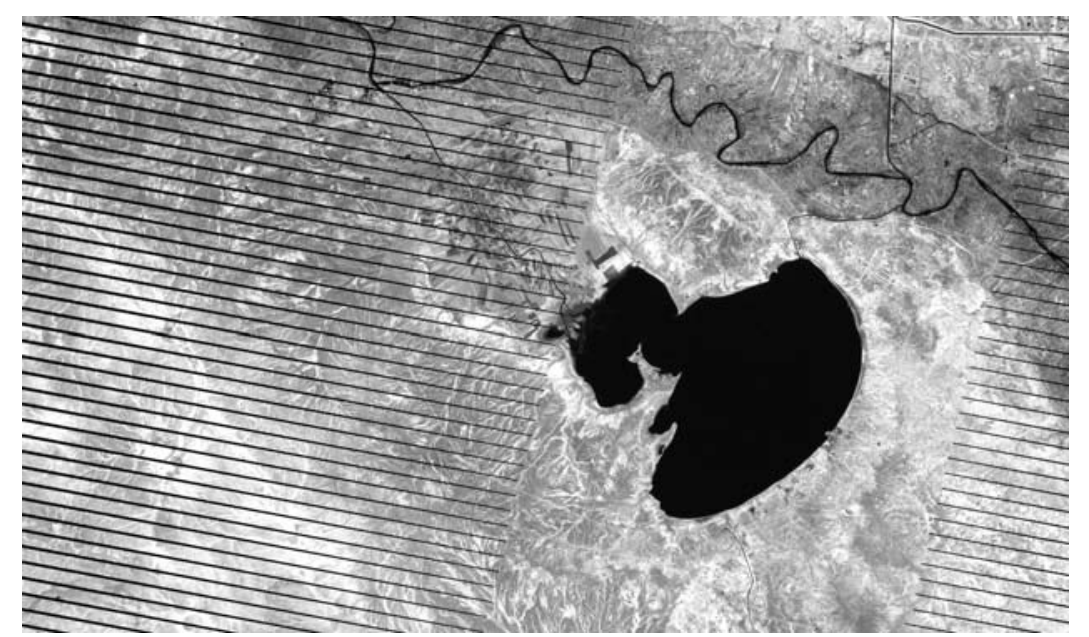

Fig.2: The image contains a scan line corrector (SLC) taken from USGS website (https://earthexplorer.usgs.gov/). The date of this picture is 12/11/2018 [7]. 
After viewing it, we perform the Focal Analysis option as shown in Fig.3. This dialog box lets you create a job definition by using the appropriate input objects and the available spatial magnifier functions. The result for this expression determines your output.

Median Value

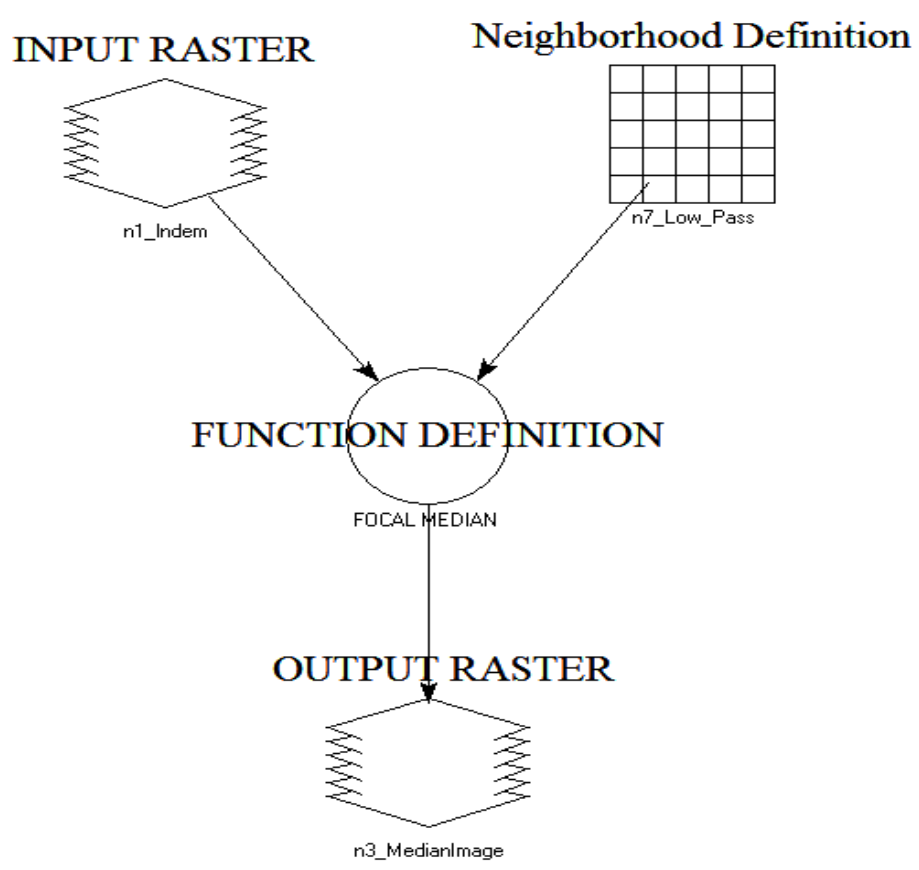

Fig.3: Describe the focal analysis process to handle Landsat images that contain scan line corrector [8].

Where, focal analysis will be applied. The black lines disappear from their ends as shown in the Fig.4.
By repeating the process for the same image, we will get a corrected image as in Fig.5.

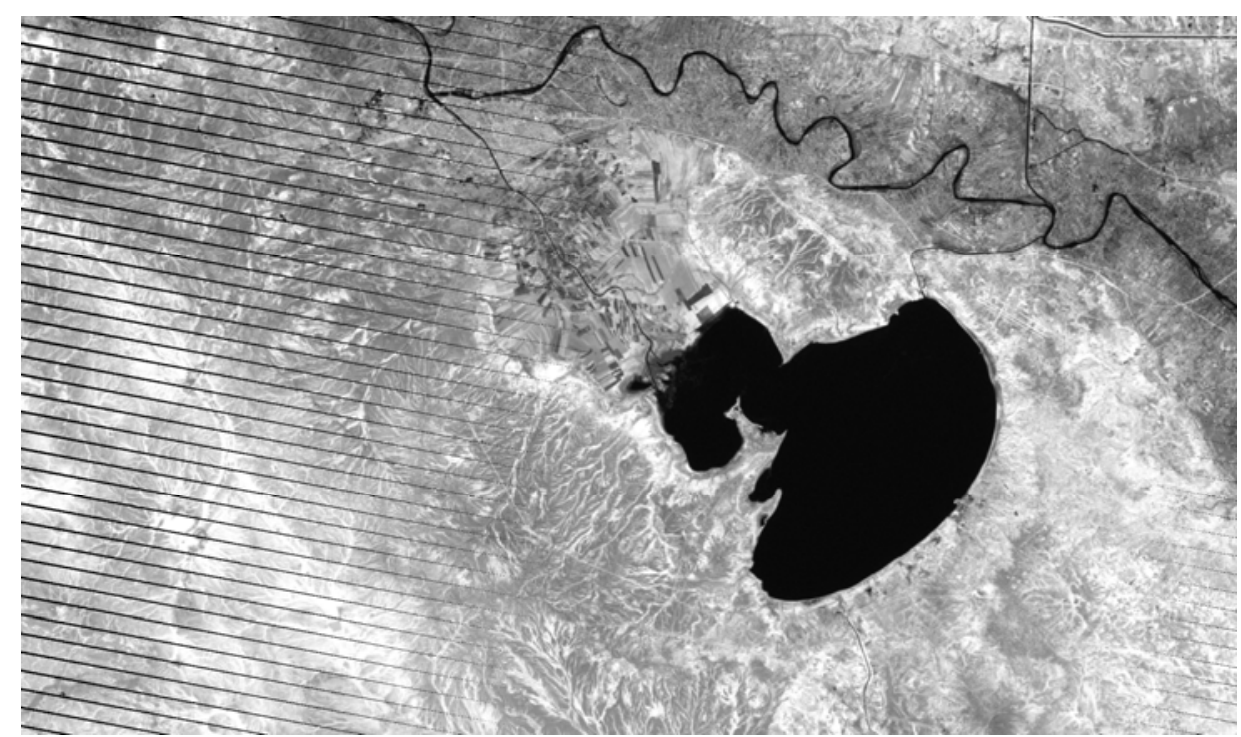

Fig.4: This format illustrates the focal analysis process when executed once. 


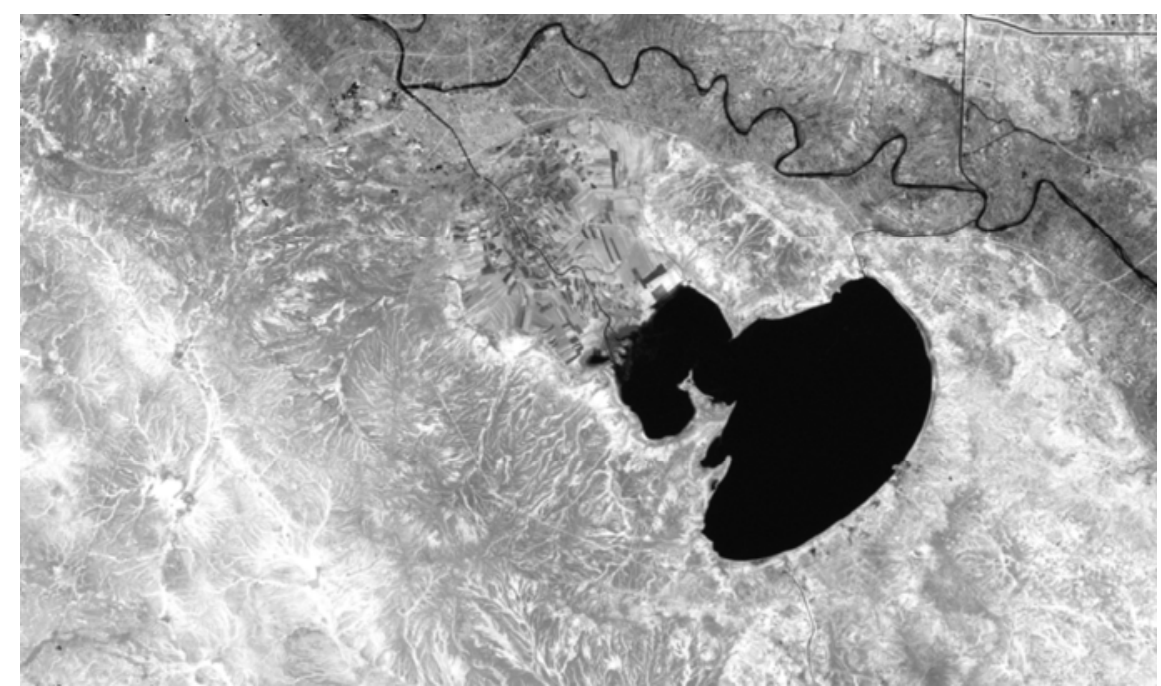

Fig.5: The image shows the corrections after repeated process of the Focal analysis more than once.

\section{Conclusion}

In this research, we correct the Scan Line Corrector (SLC) in the picture Landsat 7 and $22 \%$ of this image damaged where black lines. Images are reflected, loss and gradually decrease in the frequency of data, which are clear along the eastern and western edges of the space scene and dissolves towards the center of the space scene. Using the Erdas imagine program, we apply the focal analysis command. This applied the Median filter ( $3 \times 3)$ to the image as it corrects the Scan Line Corrector.

\section{References}

[1] K. Konstantinides and V. Bhaskaram, IEEE Computer Graphicsand Application, 12, 6 (1996) 75-86.

[2] W. Wang, M. N. S. Swamy, M. O. Ahmad, 4th IEEE International Workshop on System-on-chip for Real-time Application, (2004) 77-80.
[3] Weyori, Benjammin Asubam., "Improved Median Filtring Algorithm For The Reduction of Impulse Noise in Corrupted 2D Greyscale Images" Msc. Thesis, College of Engineering, (2011).

[4] Kwame Osei Boateng, Benjamin Weyori Asubam, David Sanka Laar., International Journal of Electronics and Communication Engineering, 5, 1 (2012) 85-97.

[5] T. Arvidson, J. Gaschand, S.N. Goward, Remote Sensing of Environment, 78, (1-2) (2001) 13-26.

[6] Pratt, William K., "Digital Image Processing". New York, John Wiley \& Sons, Inc, (1991).

[7]USGS, (https://earthexplorer.usgs.gov).

[8] Erdas Imagine 2013 Software. 